\title{
Speech Perception Performance of Native Speakers of Marathi: Effect of Filtered Speech Stimulus and Degree of Hearing Impairment
}

\author{
S. B. Rathna Kumar \\ Ali Yavar Jung National Institute of Speech \& Hearing Disabilities (Divyangjan), Mumbai, India \\ Niharika Dash \\ Institute of Health Science, Bhubaneshwar, India \\ Mendem Bapuji \\ MAA Institute of Speech \& Hearing, Hyderabad, Telangana, India \\ S. Arulmozi \\ Center for Applied Linguistics \& Translation Studies, University of Hyderabad, India \\ Chandrahas Chandanshive \\ B. Y. L. Nair Charitable Hospital \& Topiwala National Medical College, Mumbai, India
}

\begin{abstract}
The study investigated the effect of filtered speech stimulus on speech perception performance of native speakers of Marathi as a function of degree of hearing impairment. Speech identification score (SIS) testing was performed to measure speech perception on three groups (Group I, Group II, and Group III consisted of participants with moderate, moderately-severe, and severe sensorineural hearing impairment respectively). Speech stimuli comprised eight word-lists with each list consisting of 25 words in Marathi. The first seven word-lists (first to seven) were filtered at $500 \mathrm{~Hz}, 1000 \mathrm{~Hz}, 1500 \mathrm{~Hz}, 2000 \mathrm{~Hz}, 2500 \mathrm{~Hz}, 3000 \mathrm{~Hz}$, and $3500 \mathrm{~Hz}$ cut-off frequencies, respectively, while word list 8 was left unfiltered. Although, the SIS improved with increase in cut-off frequency, the improvement in SIS with increase in cut-off frequency of speech stimulus was noticed up to $3000 \mathrm{~Hz}, 2500 \mathrm{~Hz}$, and $2000 \mathrm{~Hz}$ for participants of Group I, Group II, and Group III, respectively. In addition, the improvement in speech perception performance did not correspond to what would be anticipated with an increase in the cut-off frequency of speech stimulus for participants of Group II and Group III compared to participants of Group I. Although, there was a significant reduction in SIS as a function of the degree of hearing impairment for speech stimulus filtered at $1500 \mathrm{~Hz}, 2000 \mathrm{~Hz}, 2500 \mathrm{~Hz}$, and $3000 \mathrm{~Hz}$ cut-off frequencies, there was no significant effect of degree of hearing impairment on SIS for speech stimulus filtered at $500 \mathrm{~Hz}$ and $1000 \mathrm{~Hz}$ cut-off frequencies.
\end{abstract}

Index Terms-filtered speech stimulus, degree of hearing impairment, cut-off frequency, speech identification score, Marathi

\section{INTRODUCTION}

Speech is one of the most important vehicles of human communication systems, and hearing is the building block upon which our complex communication system is designed. The perception of speech is most generally assessed in terms of an individual's auditory capability to recognize regular components of speech such as phonemes, words, and sentences (Winn, Won \& Moon, 2016). There are several spectral and temporal variations in the speech which contribute to the perception of speech signals across languages (Avilala, Prabhu \& Barman, 2010). Thus, speech perception is influenced by how psychophysical properties of speech, such as spectral and temporal features, are identified and distinguished, which is in turn influenced by a person's auditory capability (Win, Won \& Moon, 2016). A systematic study is therefore required to determine the significance of spectral and/or temporal features of speech for speech perception. The significance of spectral or temporal features to speech perception can be explored by altering one parameter while leaving another unaltered. Thus, one can study the contribution of different spectral components of speech in speech perception by manipulating the spectral properties of speech, such as the use of filtered speech stimulus (Avilala, Prabhu \& Barman, 2010). Similarly, the contribution of temporal components of speech in speech perception can be studied by manipulating the temporal characteristics of speech, such as time-compressed speech stimulus (Bhargavi, Prakash, Kumar \& Sindhura, 2011).

Bornstein, Wilson \& Cambron (1994) investigated the effect of filtered speech on speech identification performance of native speakers of English and observed that individuals with normal hearing obtained 70\% SIS for speech stimuli 
filtered at $1500 \mathrm{~Hz}$ cut-off frequency. The results of the studies on the English language cannot be generalized and applied to the Indian languages. Similarly, the research reports on the findings of one Indian language cannot be applied to another Indian language due to the differences in psychophysical characteristics of speech such as spectral energy across languages (Avilala, Prabhu \& Barman, 2010; Kumar, Patil, Saxena, Bapuji \& Chacko (2021). Subsequently, Avilala, Prabhu \& Barman (2010) investigated the effect of filtered speech on speech identification performance of native speakers of Kannada (South Indian Dravidian language) and observed that individuals with normal hearing achieved $70 \%$ SIS for Kannada speech stimulus filtered at $1200 \mathrm{~Hz}$ cut-off frequency. Similarly, Kumar, Patil, Saxena, Bapuji \& Chacko (2021) investigated the effect of spectrally modified speech on speech identification performance of native speakers of Marathi (Southern Indo-Aryan language) and reported that individuals with normal hearing achieved $90 \%$ SIS for Marathi speech stimulus filtered at $2500 \mathrm{~Hz}$ cut-off frequency. After conducting a retrospective analysis to compare with the findings of study on Kannada, it was observed that participants obtained 70\% correct SIS for speech stimuli filtered at $1500 \mathrm{~Hz}$ cut-off frequency in Marathi compared to speech stimuli filtered at $1200 \mathrm{~Hz}$ in Kannada (Kumar, Patil, Saxena, Bapuji \& Chacko, 2021). Because of the differences between Marathi and Kannada, the findings in one Indian language cannot be simply extrapolated and applied to other Indian languages.

Speech is a broadband signal with abundant spectral and temporal cues that are essential to the perception of speech signals (Turner, Souza \& Forget, 1995; Fu, Shannon \& Wang, 1998). The cochlea conducts an exquisite frequency analysis of a signal in normal hearing, breaking down its frequency components into a spatially distributed array of activity. A limited spectral resolution or little spectral information may be enough to understand speech in quiet listening environments (Shannon, Zeng, Kamath, Wygonski \& Ekelid, 1995; Smith, Delgutte \& Oxenham, 2002). Sensorineural hearing impairment occurs when the functioning of the cochlea is affected or when there is a dysfunction of the auditory nerve or higher centers in the auditory pathway. The auditory filters are often broader than normal, resulting in greater masking by background noises and echoes in reverberant environments and, in extreme conditions, even in silent anechoic environments (Summerfield, 1987). Consequently, a sensorineural hearing impairment encompasses not only the reduction in hearing sensitivity but also suprathreshold impairments such as poor spectral and temporal resolutions that degrade the perception of speech signals. Thus, reduction in the ability to resolve the frequency components of complex sounds (spectral resolution) and to process the temporal fine structure of sounds (temporal resolution) are some of the factors contributing to difficulty in understanding speech, especially under adverse listening conditions in individuals with sensorineural hearing impairment (Lorenzi, Gilbert, Carn, Garnier \& Moore, 2006).

One of the most important aspects of hearing aid fitting for those with sensorineural hearing impairment is trying to match the acoustic signal to the residual auditory area in order to make as much of the speech signal is made audible as possible. Hence, the main goal of hearing aid fitting for individuals with hearing impairment is to provide audibility of a wide range of input levels from a broad frequency spectrum (Stelmachowicz, Pittman, Hoover \& Lewis, 2004). However, while conventional hearing aids can offer satisfactory access in the low-to-mid frequencies of speech spectrum, they provide insufficient gain to the speech spectrum in the higher frequency range for those with severe hearing impairment and beyond (Boothroyd, 2008). Therefore, there is a tremendous need to understand the contribution of spectral energy to speech perception and to ascertain the effective cut-off frequency of speech needed for achieving maximum speech perception performance for individuals with sensorineural hearing impairment in their concerned language. The goal of this study was, therefore, to investigate the effect of filtered speech on speech perception performance of native speakers of Marathi as a function of the degree of hearing impairment. The findings of such studies would be valuable in developing better evaluation tools and management strategies for individuals with sensorineural hearing impairment.

\section{MATERIALS AND METHODS}

The study constituted an analytical research design with a purposive sampling technique. The SIS testing was performed as a measure of speech perception using a filtered speech test on native speakers of Marathi to assess the effect of filtered speech stimulus on speech perception performance as a function of the degree of hearing impairment.

\section{A. Participants}

A total of 60 individuals with post-lingual bilateral symmetrical sensorineural hearing impairment served as participants in the present study. The participants were in the age range between 38 and 55 years $(\mathrm{M}=45.80 ; \mathrm{SD}= \pm 5.64)$. They were equally divided into three groups based on the degree of hearing impairment. Group I, Group II, and Group III consisted of participants with moderate, moderately-severe, severe sensorineural hearing impairment, respectively. All the participants had 'A' type tympanogram indicative of normal middle ear condition. All the participants were native speakers of Marathi (Southern Indo-Aryan language) belonging to different regions of Maharashtra, a state of west India.

\section{B. Test Stimulus}

The filtered speech test in Marathi developed by Kumar, Patil, Saxena, Bapuji \& Chacko (2020) was applied as a stimulus to fulfill the aim of the study. The filtered speech test was established by adapting the conventional speech 
identification test in Marathi developed by Kumar, Mohanty, Ujawane \& Huzurbazar (2016). The filtered speech test consists of eight word-lists, each of which has 25 words in Marathi. The word lists 1, 2, 3, 4, 5, 6, and 7 filtered at cutoff frequencies of $500 \mathrm{~Hz}, 1000 \mathrm{~Hz}, 1500 \mathrm{~Hz}, 2000 \mathrm{~Hz}, 2500 \mathrm{~Hz}, 3000 \mathrm{~Hz}$, and $3500 \mathrm{~Hz}$ respectively, while word list 8 was left unfiltered (Kumar, Patil, Saxena, Bapuji \& Chacko, 2020).

\section{Procedure}

All of the investigations have been carried out in a double-room suite that was air-conditioned and had ambient noise levels that were within permissible limits. The audiological assessments such as pure-tone audiometry and tympanometry were performed in order to confirm that the participants were suitable for the study. The SIS testing was performed as a measure of speech perception on three groups of participants using eight word-lists. The stimulus was delivered by TDH-39 headphones through a laptop that was routed through a calibrated digital diagnostic audiometer. All the participants were tested monaurally at the most comfortable level using eight word-lists. The selection of ears for performing SIS testing was made on a random basis. An open-set response in the form of an oral response was obtained. In order to familiarize the participants with test procedure, ten practice items were presented at first.

\section{Scoring}

A score of 1 was assigned to each correct response, while a score of 0 was assigned to each incorrect response. The SIS $(\%)$ is calculated by dividing the number of words correctly repeated divided by the total number of words presented, and then multiplying the value by 100 . The SIS (\%) was then determined for each participant separately for each word list for further evaluation.

\section{E. Statistical Analysis}

The mean and standard deviation SIS (\%) values were calculated for eight word-lists obtained by the participants of three groups. One-way repeated measures ANOVA with LSD posthoc analysis was performed to determine whether there was a significant difference in mean SIS (\%) between eight word-lists for each group and three groups for each word list.

\section{RESULTS}

\section{A. Results}

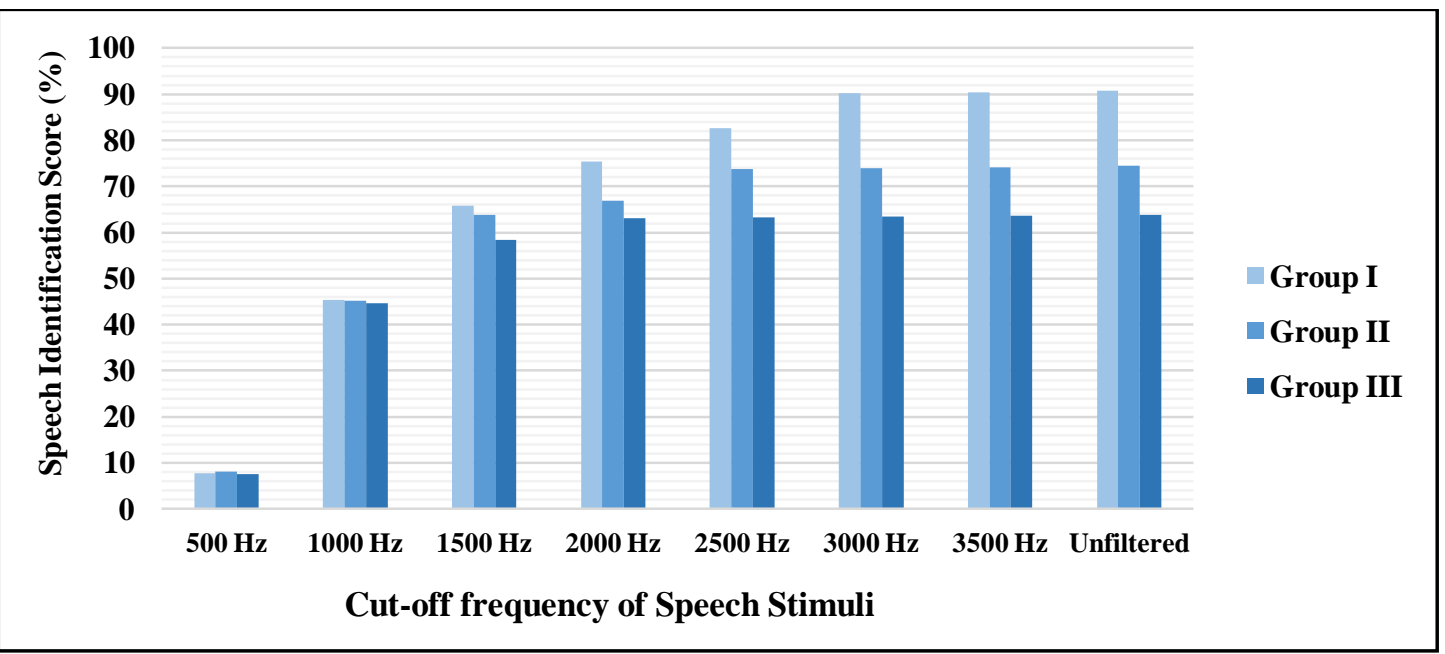

Figure 1. Comparison of mean SIS (\%) between participants of three groups for each word list 


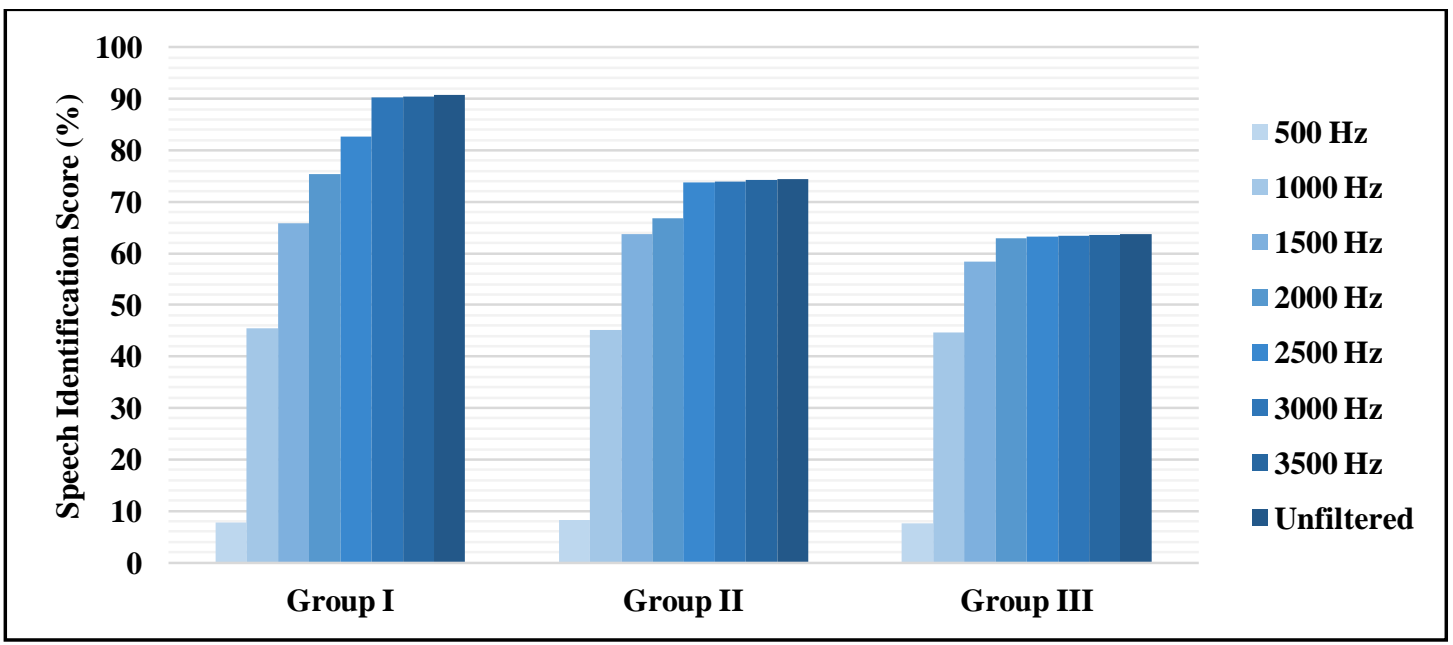

Figure 2. Comparison of mean SIS (\%) between eight word-lists for participants of each group

Figures 1 and 2 show mean SIS (\%) values between three groups for each word list and between eight word-lists for each group, respectively. One-way repeated measures of ANOVA revealed no statistically significant difference ( $>>0.05$ ) in mean SIS (\%) between and within groups for speech stimulus filtered at $500 \mathrm{~Hz}$ and $1000 \mathrm{~Hz}$ cut-off frequencies. However, there was a statistically significant difference $(\mathrm{p}<0.05)$ in mean SIS $(\%)$ between and within groups for speech stimulus filtered at $1500 \mathrm{~Hz}, 2000 \mathrm{~Hz}, 2500 \mathrm{~Hz}, 3000 \mathrm{~Hz}, 3500 \mathrm{~Hz}$, and unfiltered speech stimulus (Table 1). Similarly, the difference in mean SIS (\%) between and within eight word-lists for each group was statistically significant $(\mathrm{p}<0.05)$ (Table 2$)$.

TABLE 1

\begin{tabular}{|c|c|c|c|c|c|c|}
\hline Stimuli & Groups & Sum of Squares & $\mathrm{df}$ & Mean Square & $\mathrm{F}$ & Sig. \\
\hline \multirow{3}{*}{$500 \mathrm{~Hz}$} & Between Groups & 3.733000 & 2 & 1.86700 & 0.1520 & 0.859 \\
\hline & Within Groups & 699.2000 & 57 & 12.2670 & & \\
\hline & Total & 702.9330 & 59 & & & \\
\hline \multirow[t]{3}{*}{$1000 \mathrm{~Hz}$} & Between Groups & 6.933000 & 2 & 3.46700 & 0.0870 & 0.916 \\
\hline & Within Groups & 2260.800 & 57 & 39.6630 & & \\
\hline & Total & 2267.733 & 59 & & & \\
\hline \multirow[t]{3}{*}{$1500 \mathrm{~Hz}$} & Between Groups & 615.4400 & 2 & 307.720 & 5.4250 & 0.067 \\
\hline & Within Groups & 3403.200 & 60 & 56.7200 & & \\
\hline & Total & 4018.640 & 62 & & & \\
\hline \multirow[t]{3}{*}{$2000 \mathrm{~Hz}$} & Between Groups & 1658.133 & 2 & 829.067 & 16.882 & 0.000 \\
\hline & Within Groups & 2799.200 & 57 & 49.1090 & & \\
\hline & Total & 4457.333 & 59 & & & \\
\hline \multirow[t]{3}{*}{$2500 \mathrm{~Hz}$} & Between Groups & 3774.400 & 2 & 1887.20 & 52.239 & 0.000 \\
\hline & Within Groups & 2059.200 & 57 & 36.1260 & & \\
\hline & Total & 5833.600 & 59 & & & \\
\hline \multirow[t]{3}{*}{$3000 \mathrm{~Hz}$} & Between Groups & 7286.933 & 2 & 3643.46 & 137.353 & 0.000 \\
\hline & Within Groups & 1512.000 & 57 & 26.5260 & & \\
\hline & Total & 8798.933 & 59 & & & \\
\hline \multirow[t]{3}{*}{$3500 \mathrm{~Hz}$} & Between Groups & 7286.933 & 2 & 3643.46 & 151.722 & 0.000 \\
\hline & Within Groups & 1368.800 & 57 & 24.0140 & & \\
\hline & Total & 8655.733 & 59 & & & \\
\hline \multirow[t]{3}{*}{ Unfiltered } & Between Groups & 7402.133 & 2 & 3701.06 & 164.916 & 0.000 \\
\hline & Within Groups & 1279.200 & 57 & 22.4420 & & \\
\hline & Total & 8681.333 & 59 & & & \\
\hline
\end{tabular}

TABLE 2

ONE-WAy ANOVA Results SHOWING THE COMPARISON OF MEAN SIS (\%) BETWEen AND WiTHIN SPEECH STIMULI FoR EACH GROUP

\begin{tabular}{|c|c|c|c|c|c|c|}
\hline Groups & Speech Stimuli & Sum of Squares & df & Mean Square & $\mathrm{F}$ & Sig. \\
\hline \multirow[t]{3}{*}{ Group I } & Between Stimuli & 118391.600 & 7 & 16913.086 & 926.077 & 0.000 \\
\hline & Within Stimuli & 2776.000 & 152 & 18.263000 & & \\
\hline & Total & 121167.600 & 159 & & & \\
\hline \multirow[t]{3}{*}{ Group II } & Between Stimuli & 75167.600 & 7 & 10738.229 & 252.508 & 0.000 \\
\hline & Within Stimuli & 6464.000 & 152 & 42.526000 & & \\
\hline & Total & 81631.600 & 159 & & & \\
\hline \multirow[t]{3}{*}{ Group III } & Between Stimuli & 56709.660 & 7 & 8101.3800 & 213.531 & 0.000 \\
\hline & Within Stimuli & 6070.400 & 160 & 37.940000 & & \\
\hline & Total & 62780.060 & 167 & & & \\
\hline
\end{tabular}


The data were further subjected to LSD post-hoc multiple comparisons and the results were discussed under the following headings: Comparison of mean SIS (\%) between three groups for each filtered speech stimulus; Comparison of mean SIS $(\%)$ between different filtered speech stimuli for participants with moderate sensorineural hearing impairment (Group I); Comparison of mean SIS (\%) between different filtered speech stimuli for participants with moderately-severe sensorineural hearing impairment (Group II); Comparison of mean SIS (\%) between different filtered speech stimuli for participants with severe sensorineural hearing impairment (Group III).

\section{Comparison of Mean SIS (\%) between Three Groups for Each Filtered Speech Stimulus and Unfiltered Speech Stimulus}

LSD post-hoc multiple comparison revealed no significant difference ( $p>0.05)$ in mean SIS (\%) between participants with moderate hearing impairment (Group I) and moderately-severe hearing impairment (Group II), moderate hearing impairment (Group I) and severe hearing impairment (Group III), and moderately-severe hearing impairment (Group II) and severe hearing impairment (Group III) for speech stimuli filtered at $500 \mathrm{~Hz}$ and $1000 \mathrm{~Hz}$ cut-off frequencies. However, a statistically significant difference $(\mathrm{p}<0.05)$ in mean SIS $(\%)$ was noticed between participants with moderate hearing impairment (Group I) and moderately-severe hearing impairment (Group II), moderate hearing impairment (Group I) and severe hearing impairment (Group III), and moderately-severe hearing impairment (Group II) and severe hearing impairment (Group III) for speech stimuli filtered at $1500 \mathrm{~Hz}, 2000 \mathrm{~Hz}, 2500 \mathrm{~Hz}, 3000 \mathrm{~Hz}, 3500 \mathrm{~Hz}$ cut-off frequencies, and unfiltered speech stimulus (Table 3).

TABLE 3

LSD POST-Hoc Multiple COMParison Of MEAN SIS (\%) AMONG THREE GROUPS For EACH SPEECH STIMULUS

\begin{tabular}{|c|c|c|c|c|c|c|c|}
\hline \multirow[t]{2}{*}{ Stimuli } & \multicolumn{2}{|l|}{ Groups } & \multirow{2}{*}{$\begin{array}{l}\text { Mean Difference (I- } \\
\text { J) }\end{array}$} & \multirow{2}{*}{$\begin{array}{l}\text { Standard } \\
\text { Error }\end{array}$} & \multirow[t]{2}{*}{ Sig. } & \multicolumn{2}{|l|}{$95 \% \mathrm{CI}$} \\
\hline & I & $\mathrm{J}$ & & & & Lower & Upper \\
\hline \multirow{2}{*}{$500 \mathrm{~Hz}$} & Group I & Group II & -0.400000 & 1.107550 & 0.719 & -2.61783 & 1.817830 \\
\hline & & Group III & 0.200000 & 1.107550 & 0.857 & -2.01783 & 2.417830 \\
\hline \multirow{3}{*}{$1000 \mathrm{~Hz}$} & Group I & Group II & 0.200000 & 1.991561 & 0.920 & -3.78803 & 4.188030 \\
\hline & & Group III & 0.800000 & 1.991561 & 0.689 & -3.18803 & 4.788030 \\
\hline & Group II & Group III & 0.600000 & 1.991561 & 0.764 & -3.38803 & 4.588030 \\
\hline \multirow{2}{*}{$1500 \mathrm{~Hz}$} & & Group III & $7.400000^{*}$ & 2.324200 & 0.002 & 2.750910 & 12.04909 \\
\hline & Group II & Group III & $5.400000^{*}$ & 2.324200 & 0.024 & 0.750910 & 10.04909 \\
\hline \multirow[t]{3}{*}{$2000 \mathrm{~Hz}$} & Group I & Group II & $8.600000^{*}$ & 2.216050 & 0.000 & 4.162440 & 13.03756 \\
\hline & & Group III & $12.60000^{*}$ & 2.216050 & 0.000 & 8.162440 & 17.03756 \\
\hline & Group II & Group III & $4.000000^{*}$ & 2.216050 & 0.046 & -.437560 & 8.437560 \\
\hline $2500 \mathrm{~Hz}$ & Group I & Group II & $8.800000 *$ & 1.900692 & 0.000 & 4.993930 & 12.60607 \\
\hline $3000 \mathrm{~Hz}$ & Group II & Group III & $10.60000^{*}$ & 1.628690 & 0.000 & 7.338600 & 13.86140 \\
\hline \multirow[t]{3}{*}{$3500 \mathrm{~Hz}$} & Group I & Group II & $16.20000^{*}$ & 1.549646 & 0.000 & 13.09689 & 19.30311 \\
\hline & & Group III & $26.80000^{*}$ & 1.549646 & 0.000 & 23.69689 & 29.90311 \\
\hline & Group II & Group III & $10.60000^{*}$ & 1.549646 & 0.000 & 7.496890 & 13.70311 \\
\hline \multirow[t]{3}{*}{ Unfiltered } & Group I & Group II & $16.40000 *$ & 1.498069 & 0.000 & 13.40017 & 19.39983 \\
\hline & & Group III & $27.00000 *$ & 1.498069 & 0.000 & 24.00017 & 29.99983 \\
\hline & Group II & Group III & $10.60000 *$ & 1.498069 & 0.000 & 7.600170 & 13.59983 \\
\hline
\end{tabular}

* The mean difference is significant at the 0.05 level

\section{Comparison of Mean SIS (\%) between Different Filtered Speech Stimuli for Participants with Moderate Sensorineural Hearing Impairment (Group I)}

LSD post-hoc multiple comparison in participants with moderate sensorineural hearing impairment (Group I) showed significant difference in mean SIS (\%) between speech stimulus filtered at $500 \mathrm{~Hz}$ cut-off frequency and speech stimuli filtered at $1000 \mathrm{~Hz}, 1500 \mathrm{~Hz}, 2000 \mathrm{~Hz}, 2500 \mathrm{~Hz}, 3000 \mathrm{~Hz}, 3500 \mathrm{~Hz}$, and unfiltered speech stimulus; speech stimulus filtered at $1000 \mathrm{~Hz}$ cut-off frequency and speech stimuli filtered at $1500 \mathrm{~Hz}, 2000 \mathrm{~Hz}, 2500 \mathrm{~Hz}, 3000 \mathrm{~Hz}, 3500 \mathrm{~Hz}$ cutoff frequencies, and unfiltered speech stimulus; speech stimulus filtered at $1500 \mathrm{~Hz}$ cut-off frequency and speech stimuli filtered at $2000 \mathrm{~Hz}, 2500 \mathrm{~Hz}, 3000 \mathrm{~Hz}, 3500 \mathrm{~Hz}$ cut-off frequencies, and unfiltered speech stimulus; speech stimulus filtered at $2000 \mathrm{~Hz}$ cut-off frequency and speech stimuli filtered at $2500 \mathrm{~Hz}, 3000 \mathrm{~Hz}, 3500 \mathrm{~Hz}$ cut-off frequencies, and unfiltered speech stimulus; speech stimulus filtered at $2500 \mathrm{~Hz}$ cut-off frequency and speech stimuli filtered at $3000 \mathrm{~Hz}, 3500 \mathrm{~Hz}$ cut-off frequencies, and unfiltered speech stimulus. However, there was no significant difference SIS (\%) between speech stimulus filtered at $3000 \mathrm{~Hz}$ cut-off frequency and speech stimulus filtered at 3500 $\mathrm{Hz}$ cut-off frequency, and unfiltered speech stimulus; speech stimulus filtered at $3500 \mathrm{~Hz}$ cut-off frequency and unfiltered speech stimulus (Table 4). 
TABLE 4

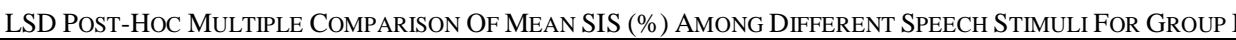

\begin{tabular}{|c|c|c|c|c|c|c|}
\hline \multicolumn{2}{|c|}{ Speech Stimuli } & \multirow{2}{*}{$\begin{array}{l}\text { Mean Difference (I- } \\
\text { J) }\end{array}$} & \multirow{2}{*}{ Standard Error } & \multirow[t]{2}{*}{ Sig. } & \multicolumn{2}{|c|}{ 95\% Confidence Interval } \\
\hline $\mathrm{I}$ & $\mathrm{J}$ & & & & Lower & Upper \\
\hline \multirow{7}{*}{$500 \mathrm{~Hz}$} & $1000 \mathrm{~Hz}$ & $-37.600000^{*}$ & 1.351413 & 0.000 & -40.26998 & -34.93002 \\
\hline & $1500 \mathrm{~Hz}$ & $-58.000000 *$ & 1.351413 & 0.000 & -60.66998 & -55.33002 \\
\hline & $2000 \mathrm{~Hz}$ & $-67.600000 *$ & 1.351413 & 0.000 & -70.26998 & -64.93002 \\
\hline & $2500 \mathrm{~Hz}$ & $-74.800000 *$ & 1.351413 & 0.000 & -77.46998 & -72.13002 \\
\hline & $3000 \mathrm{~Hz}$ & $-82.400000^{*}$ & 1.351413 & 0.000 & -85.06998 & -79.73002 \\
\hline & $3500 \mathrm{~Hz}$ & $-82.600000^{*}$ & 1.351413 & 0.000 & -85.26998 & -79.93002 \\
\hline & Unfiltered & $-83.000000 *$ & 1.351413 & 0.000 & -85.66998 & -80.33002 \\
\hline \multirow[t]{6}{*}{$1000 \mathrm{~Hz}$} & $1500 \mathrm{~Hz}$ & $-20.400000 *$ & 1.351413 & 0.000 & -23.06998 & -17.73002 \\
\hline & $2000 \mathrm{~Hz}$ & $-30.000000 *$ & 1.351413 & 0.000 & -32.66998 & -27.33002 \\
\hline & $2500 \mathrm{~Hz}$ & $-37.200000 *$ & 1.351413 & 0.000 & -39.86998 & -34.53002 \\
\hline & $3000 \mathrm{~Hz}$ & $-44.800000^{*}$ & 1.351413 & 0.000 & -47.46998 & -42.13002 \\
\hline & $3500 \mathrm{~Hz}$ & $-45.000000 *$ & 1.351413 & 0.000 & -47.66998 & -42.33002 \\
\hline & Unfiltered & $-45.400000^{*}$ & 1.351413 & 0.000 & -48.06998 & -42.73002 \\
\hline \multirow[t]{5}{*}{$1500 \mathrm{~Hz}$} & $2000 \mathrm{~Hz}$ & $-9.600000 *$ & 1.351413 & 0.000 & -12.26998 & -6.93002 \\
\hline & $2500 \mathrm{~Hz}$ & $-16.800000^{*}$ & 1.351413 & 0.000 & -19.46998 & -14.13002 \\
\hline & $3000 \mathrm{~Hz}$ & $-24.400000^{*}$ & 1.351413 & 0.000 & -27.06998 & -21.73002 \\
\hline & $3500 \mathrm{~Hz}$ & $-24.600000 *$ & 1.351413 & 0.000 & -27.26998 & -21.93002 \\
\hline & Unfiltered & $-25.000000 *$ & 1.351413 & 0.000 & -27.66998 & -22.33002 \\
\hline \multirow[t]{4}{*}{$2000 \mathrm{~Hz}$} & $2500 \mathrm{~Hz}$ & $-7.200000 *$ & 1.351413 & 0.000 & -9.86998 & -4.53002 \\
\hline & $3000 \mathrm{~Hz}$ & $-14.800000^{*}$ & 1.351413 & 0.000 & -17.46998 & -12.13002 \\
\hline & $3500 \mathrm{~Hz}$ & $-15.000000^{*}$ & 1.351413 & 0.000 & -17.66998 & -12.33002 \\
\hline & Unfiltered & $-15.400000^{*}$ & 1.351413 & 0.000 & -18.06998 & -12.73002 \\
\hline \multirow[t]{3}{*}{$2500 \mathrm{~Hz}$} & $3000 \mathrm{~Hz}$ & $-7.600000 *$ & 1.351413 & 0.000 & -10.26998 & -4.930020 \\
\hline & $3500 \mathrm{~Hz}$ & $-7.800000 *$ & 1.351413 & 0.000 & -10.46998 & -5.130020 \\
\hline & Unfiltered & $-8.200000^{*}$ & 1.351413 & 0.000 & -10.86998 & -5.530020 \\
\hline \multirow[t]{2}{*}{$3000 \mathrm{~Hz}$} & $3500 \mathrm{~Hz}$ & -.2000000 & 1.351413 & 0.883 & -2.869980 & 2.469980 \\
\hline & Unfiltered & -.6000000 & 1.351413 & 0.658 & -3.269980 & 2.069980 \\
\hline $3500 \mathrm{~Hz}$ & Unfiltered & -.4000000 & 1.351413 & 0.768 & -3.069980 & 2.269980 \\
\hline
\end{tabular}

"The mean difference is significant at the 0.05 level

\section{Comparison of Mean SIS (\%) between Different Filtered Speech Stimuli for Participants with Moderately-Severe Sensorineural Hearing Impairment (Group II)}

LSD post-hoc multiple comparison for participants with moderately-severe sensorineural hearing impairment (Group II) showed significant difference in SIS (\%) between speech stimulus filtered at $500 \mathrm{~Hz}$ cut-off frequency and speech stimuli filtered at $1000 \mathrm{~Hz}, 1500 \mathrm{~Hz}, 2000 \mathrm{~Hz}, 2500 \mathrm{~Hz}, 3000 \mathrm{~Hz}, 3500 \mathrm{~Hz}$, and unfiltered speech stimulus; speech stimulus filtered at $1000 \mathrm{~Hz}$ cut-off frequency and speech stimuli filtered at $1500 \mathrm{~Hz}, 2000 \mathrm{~Hz}, 2500 \mathrm{~Hz}, 3000 \mathrm{~Hz}, 3500$ $\mathrm{Hz}$ cut-off frequencies, and unfiltered speech stimulus; speech stimulus filtered at $1500 \mathrm{~Hz}$ cut-off frequency and speech stimuli filtered at $2000 \mathrm{~Hz}, 2500 \mathrm{~Hz}, 3000 \mathrm{~Hz}, 3500 \mathrm{~Hz}$ cut-off frequencies, and unfiltered speech stimulus; speech stimulus filtered at $2000 \mathrm{~Hz}$ cut-off frequency and speech stimuli filtered at $2500 \mathrm{~Hz}, 3000 \mathrm{~Hz}, 3500 \mathrm{~Hz}$ cut-off frequencies, and unfiltered speech stimulus. However, there was no significant difference in SIS (\%) between speech stimulus filtered at $2500 \mathrm{~Hz}$ cut-off frequency and speech stimuli filtered at $3000 \mathrm{~Hz}, 3500 \mathrm{~Hz}$ cut-off frequency, and unfiltered speech stimulus; speech stimulus filtered at $3000 \mathrm{~Hz}$ cut-off frequency and speech stimuli filtered at $3500 \mathrm{~Hz}$ cut-off frequency, and unfiltered speech stimulus; speech stimulus filtered at $3500 \mathrm{~Hz}$ and unfiltered speech stimulus (Table 5). 
TABLE 5

LSD Post-Hoc MUltiPle COMPaRISON OF MEAN SIS (\%) AMONG DifFERENT SPEECH STIMULi FOR GROUP II

\begin{tabular}{|c|c|c|c|c|c|c|}
\hline \multicolumn{2}{|c|}{ Speech Stimuli } & \multirow[t]{2}{*}{ Mean Difference (I-J) } & \multirow{2}{*}{$\begin{array}{l}\text { Standard } \\
\text { Error }\end{array}$} & \multirow[t]{2}{*}{ Sig. } & \multicolumn{2}{|c|}{$95 \%$ Confidence Interval } \\
\hline I & $\mathrm{J}$ & & & & Lower & Upper \\
\hline \multirow[t]{7}{*}{$500 \mathrm{~Hz}$} & $1000 \mathrm{~Hz}$ & $-37.000000^{*}$ & 2.062191 & 0.000 & -41.07426 & -32.92574 \\
\hline & $1500 \mathrm{~Hz}$ & $-55.600000 *$ & 2.062191 & 0.000 & -59.67426 & -51.52574 \\
\hline & $2000 \mathrm{~Hz}$ & $-58.600000^{*}$ & 2.062191 & 0.000 & -62.67426 & -54.52574 \\
\hline & $2500 \mathrm{~Hz}$ & $-65.600000^{*}$ & 2.062191 & 0.000 & -69.67426 & -61.52574 \\
\hline & $3000 \mathrm{~Hz}$ & $-65.800000^{*}$ & 2.062191 & 0.000 & -69.87426 & -61.72574 \\
\hline & $3500 \mathrm{~Hz}$ & $-66.000000^{*}$ & 2.062191 & 0.000 & -70.07426 & -61.92574 \\
\hline & Unfiltered & $-66.200000^{*}$ & 2.062191 & 0.000 & -70.27426 & -62.12574 \\
\hline \multirow[t]{6}{*}{$1000 \mathrm{~Hz}$} & $1500 \mathrm{~Hz}$ & $-18.600000^{*}$ & 2.062191 & 0.000 & -22.67426 & -14.52574 \\
\hline & $2000 \mathrm{~Hz}$ & $-21.600000^{*}$ & 2.062191 & 0.000 & -25.67426 & -17.52574 \\
\hline & $2500 \mathrm{~Hz}$ & $-28.600000^{*}$ & 2.062191 & 0.000 & -32.67426 & -24.52574 \\
\hline & $3000 \mathrm{~Hz}$ & $-28.800000^{*}$ & 2.062191 & 0.000 & -32.87426 & -24.72574 \\
\hline & $3500 \mathrm{~Hz}$ & $-29.000000^{*}$ & 2.062191 & 0.000 & -33.07426 & -24.92574 \\
\hline & Unfiltered & $-29.200000^{*}$ & 2.062191 & 0.000 & -33.27426 & -25.12574 \\
\hline \multirow[t]{5}{*}{$1500 \mathrm{~Hz}$} & $2000 \mathrm{~Hz}$ & -3.000000 & 2.062191 & 0.148 & -7.07426 & 1.074260 \\
\hline & $2500 \mathrm{~Hz}$ & $-10.000000^{*}$ & 2.062191 & 0.000 & -14.07426 & -5.925740 \\
\hline & $3000 \mathrm{~Hz}$ & $-10.200000^{*}$ & 2.062191 & 0.000 & -14.27426 & -6.125740 \\
\hline & $3500 \mathrm{~Hz}$ & $-10.400000^{*}$ & 2.062191 & 0.000 & -14.47426 & -6.325740 \\
\hline & Unfiltered & $-10.600000^{*}$ & 2.062191 & 0.000 & -14.67426 & -6.525740 \\
\hline \multirow[t]{4}{*}{$2000 \mathrm{~Hz}$} & $2500 \mathrm{~Hz}$ & $-7.000000 *$ & 2.062191 & 0.001 & -11.07426 & -2.925740 \\
\hline & $3000 \mathrm{~Hz}$ & $-7.200000^{*}$ & 2.062191 & 0.001 & -11.27426 & -3.125740 \\
\hline & $3500 \mathrm{~Hz}$ & $-7.400000 *$ & 2.062191 & 0.000 & -11.47426 & -3.325740 \\
\hline & Unfiltered & $-7.600000^{*}$ & 2.062191 & 0.000 & -11.67426 & -3.525740 \\
\hline \multirow{3}{*}{$2500 \mathrm{~Hz}$} & $3000 \mathrm{~Hz}$ & -2000000 & 2.062191 & 0.923 & -4.274260 & 3.874260 \\
\hline & $3500 \mathrm{~Hz}$ & -.4000000 & 2.062191 & 0.846 & -4.474260 & 3.674260 \\
\hline & Unfiltered & -.6000000 & 2.062191 & 0.771 & -4.674260 & 3.474260 \\
\hline \multirow[t]{2}{*}{$3000 \mathrm{~Hz}$} & $3500 \mathrm{~Hz}$ & -.2000000 & 2.062191 & 0.923 & -4.274260 & 3.874260 \\
\hline & Unfiltered & -.4000000 & 2.062191 & 0.846 & -4.474260 & 3.674260 \\
\hline $3500 \mathrm{~Hz}$ & Unfiltered & -.2000000 & 2.062191 & 0.923 & -4.274260 & 3.874260 \\
\hline
\end{tabular}

${ }^{*}$ The mean difference is significant at the 0.05 level

4. Comparison of Mean SIS (\%) between Different Filtered Speech Stimuli for Participants with Severe Sensorineural Hearing Impairment (Group III)

LSD post-hoc multiple comparison for participants with severe sensorineural hearing impairment (Group III) showed significant difference in SIS (\%) between speech stimulus filtered at $500 \mathrm{~Hz}$ cut-off frequency and speech stimuli filtered at $1000 \mathrm{~Hz}, 1500 \mathrm{~Hz}, 2000 \mathrm{~Hz}, 2500 \mathrm{~Hz}, 3000 \mathrm{~Hz}, 3500 \mathrm{~Hz}$, and unfiltered speech stimulus; speech stimulus filtered at $1000 \mathrm{~Hz}$ cut-off frequency and speech stimuli filtered at $1500 \mathrm{~Hz}, 2000 \mathrm{~Hz}, 2500 \mathrm{~Hz}, 3000 \mathrm{~Hz}, 3500 \mathrm{~Hz}$ cutoff frequencies, and unfiltered speech stimulus; speech stimulus filtered at $1500 \mathrm{~Hz}$ cut-off frequency and speech stimuli filtered at $2000 \mathrm{~Hz}, 2500 \mathrm{~Hz}, 3000 \mathrm{~Hz}, 3500 \mathrm{~Hz}$ cut-off frequencies. However, there was no significant difference SIS (\%) between speech stimulus filtered at $2000 \mathrm{~Hz}$ cut-off frequency and speech stimuli filtered at $2500 \mathrm{~Hz}$, $3000 \mathrm{~Hz}, 3500 \mathrm{~Hz}$, and unfiltered speech stimulus; speech stimulus filtered at $2500 \mathrm{~Hz}$ cut-off frequency and speech stimuli filtered at $3000 \mathrm{~Hz}, 3500 \mathrm{~Hz}$ cut-off frequencies, and unfiltered speech stimulus; speech stimulus filtered at $3000 \mathrm{~Hz}$ cut-off frequency and speech stimulus filtered at $3500 \mathrm{~Hz}$ cut-off frequency and unfiltered speech stimulus; speech stimulus filtered at $3500 \mathrm{~Hz}$ and unfiltered speech stimulus (Table 6). 
TABLE 6

LSD POST-HOC MULTIPLE COMPARISON OF MEAN SIS (\%) AMONG DifFERENT SPEECH STIMULI For GROUP III

\begin{tabular}{|c|c|c|c|c|c|c|}
\hline \multicolumn{2}{|c|}{ Speech Stimuli } & \multirow{2}{*}{$\begin{array}{l}\text { Mean Difference (I- } \\
\text { J) }\end{array}$} & \multirow[t]{2}{*}{ Standard Error } & \multirow[t]{2}{*}{ Sig. } & \multicolumn{2}{|c|}{ 95\% Confidence Interval } \\
\hline I & $\mathrm{J}$ & & & & Lower & Upper \\
\hline \multirow[t]{7}{*}{$500 \mathrm{~Hz}$} & $1000 \mathrm{~Hz}$ & $-37.000000^{*}$ & 1.900877 & 0.000 & -40.75404 & -33.24596 \\
\hline & $1500 \mathrm{~Hz}$ & $-50.800000^{*}$ & 1.900877 & 0.000 & -54.55404 & -47.04596 \\
\hline & $2000 \mathrm{~Hz}$ & $-55.400000^{*}$ & 1.900877 & 0.000 & -59.15404 & -51.64596 \\
\hline & $2500 \mathrm{~Hz}$ & $-55.600000^{*}$ & 1.900877 & 0.000 & -59.35404 & -51.84596 \\
\hline & $3000 \mathrm{~Hz}$ & $-55.800000^{*}$ & 1.900877 & 0.000 & -59.55404 & -52.04596 \\
\hline & $3500 \mathrm{~Hz}$ & $-56.000000^{*}$ & 1.900877 & 0.000 & -59.75404 & -52.24596 \\
\hline & Unfiltered & $-56.200000^{*}$ & 1.900877 & 0.000 & -59.95404 & -52.44596 \\
\hline \multirow[t]{6}{*}{$1000 \mathrm{~Hz}$} & $1500 \mathrm{~Hz}$ & $-13.800000^{*}$ & 1.900877 & 0.000 & -17.55404 & -10.04596 \\
\hline & $2000 \mathrm{~Hz}$ & $-18.400000^{*}$ & 1.900877 & 0.000 & -22.15404 & -14.64596 \\
\hline & $2500 \mathrm{~Hz}$ & $-18.600000^{*}$ & 1.900877 & 0.000 & -22.35404 & -14.84596 \\
\hline & $3000 \mathrm{~Hz}$ & $-18.800000^{*}$ & 1.900877 & 0.000 & -22.55404 & -15.04596 \\
\hline & $3500 \mathrm{~Hz}$ & $-19.000000^{*}$ & 1.900877 & 0.000 & -22.75404 & -15.24596 \\
\hline & Unfiltered & $-19.200000^{*}$ & 1.900877 & 0.000 & -22.95404 & -15.44596 \\
\hline \multirow[t]{5}{*}{$1500 \mathrm{~Hz}$} & $2000 \mathrm{~Hz}$ & $-4.600000^{*}$ & 1.900877 & 0.017 & -8.35404 & -.8459600 \\
\hline & $2500 \mathrm{~Hz}$ & $-4.800000 *$ & 1.900877 & 0.013 & -8.55404 & -1.045960 \\
\hline & $3000 \mathrm{~Hz}$ & $-5.000000 *$ & 1.900877 & 0.009 & -8.75404 & -1.245960 \\
\hline & $3500 \mathrm{~Hz}$ & $-5.200000 *$ & 1.900877 & 0.007 & -8.95404 & -1.445960 \\
\hline & Unfiltered & $-5.400000 *$ & 1.900877 & 0.005 & -9.15404 & -1.645960 \\
\hline \multirow[t]{4}{*}{$2000 \mathrm{~Hz}$} & $2500 \mathrm{~Hz}$ & -.2000000 & 1.900877 & 0.916 & -3.95404 & 3.554040 \\
\hline & $3000 \mathrm{~Hz}$ & -.4000000 & 1.900877 & 0.834 & -4.15404 & 3.354040 \\
\hline & $3500 \mathrm{~Hz}$ & -.6000000 & 1.900877 & 0.753 & -4.35404 & 3.154040 \\
\hline & Unfiltered & -8000000 & 1.900877 & 0.674 & -4.55404 & 2.954040 \\
\hline \multirow[t]{3}{*}{$2500 \mathrm{~Hz}$} & $3000 \mathrm{~Hz}$ & -.2000000 & 1.900877 & 0.916 & -3.95404 & 3.554040 \\
\hline & $3500 \mathrm{~Hz}$ & -.4000000 & 1.900877 & 0.834 & -4.15404 & 3.354040 \\
\hline & Unfiltered & -.6000000 & 1.900877 & 0.753 & -4.35404 & 3.154040 \\
\hline \multirow[t]{2}{*}{$3000 \mathrm{~Hz}$} & $3500 \mathrm{~Hz}$ & -.2000000 & 1.900877 & 0.916 & -3.95404 & 3.554040 \\
\hline & Unfiltered & -.4000000 & 1.900877 & 0.834 & -4.15404 & 3.354040 \\
\hline $3500 \mathrm{~Hz}$ & Unfiltered & -.2000000 & 1.900877 & 0.916 & -3.95404 & 3.554040 \\
\hline
\end{tabular}

The mean difference is significant at the 0.05 level

\section{DISCUSSION}

The ultimate goal of fitting hearing aids for individuals with sensorineural hearing impairment is to provide appropriate amplification across wide range of frequencies in order to make as much of the speech signal audible as possible (Stelmachowicz, Pittman, Hoover \& Lewis, 2004). However, according to studies, individuals who are provided audibility at frequencies where their hearing thresholds are severe and/or sloping do not demonstrate any improvement in speech perception due to the limited ability to utilize the amplified signal in that frequency region (Hogan \& Turner, 1998; Ching, Dillon, Katsch \& Byrne, 2001). On the other hand, few studies have reported that individuals with sloping sensorineural hearing loss have demonstrated improvements in speech understanding, especially in noisy environments when they are provided with high frequency amplification (Turner \& Henry, 2002; Mackersie, Crocker \& Davis, 2004). While most of the studies appear to support the general notion that high-frequency amplification may not always be beneficial, some studies reported otherwise. Therefore, a clearly established rule must be precluded that would distinguish individuals with hearing impairment who are likely to benefit from high-frequency amplification from those who are unlikely to benefit (Yadav, Kumar, Annapurna \& Vinila, 2011). The present study was therefore aimed to determine the ability of an individual to derive maximum speech perception performance as a function of filtered speech stimulus with respect to degree of hearing impairment.

The results revealed that there was a significant effect of filtered speech stimulus on speech perception performance. The SIS significantly improved with increase in cut-off frequency of speech stimulus up to $3000 \mathrm{~Hz}, 2500 \mathrm{~Hz}$, and 2000 $\mathrm{Hz}$ for participants moderate hearing impairment (Group I), moderately-severe hearing impairment (Group II), and severe hearing impairment (Group III) respectively and remained consistent at higher cut-off frequencies. The participants of Group I, Group II, and Group III achieved maximum SIS of 90.4\%, 74.2\%, and 63.6\% for speech stimulus filtered at $3000 \mathrm{~Hz}, 2500 \mathrm{~Hz}$, and $2000 \mathrm{~Hz}$ cut-off frequencies respectively. A loss of hearing sensitivity that increases with an increase in frequency is the most frequent type of sensorineural hearing impairment (Turner, Gantz, Lowder \& Gfeller, 2005). The improvement in speech perception with additional high-frequency information does not approximate what would have been expected based on the increase in audible high frequency information when hearing loss in the high frequency region (about $2500 \mathrm{~Hz}$ and above) is more than 60 to $80 \mathrm{~dB}$ (Pavlovic, 1984; Hogan \& Turner, 1998; Ching, Psarros, Hill, Dillon \& Incerti, 2001). The lack of improvement in speech perception with added high frequency information has been attributed to the presence of non-functioning inner hair cells in the high frequency region of the cochlea known as dead regions (Moore, Glasberg \& Baer, 1997; Vickers, Baer \& Moore, 2001; Baer, Moore \& Kluk, 2002). This limits the ability of an individual to access the speech spectrum in the higher frequency region. Hence, the participants with moderately severe and severe hearing impairment could extract spectral 
information only up to $2500 \mathrm{~Hz}$ and $2000 \mathrm{~Hz}$, respectively, as compared to participants with moderate hearing impairment who could extract spectral information up to $3000 \mathrm{~Hz}$.

There was no significant effect of degree of hearing impairment on speech perception performance for speech stimulus filtered at $500 \mathrm{~Hz}$ and $1000 \mathrm{~Hz}$ cut-off frequencies. Although, the amount of speech information that can be extracted from an audible signal decrease as the degree of hearing impairment increases, the deterioration is less severe at lower frequencies than at higher frequencies (Ching, Psarros, Hill, Dillon \& Incerti, 2001). This is because, the frequency resolution is relatively preserved in lower frequencies compared to higher frequencies, even when the degree of hearing loss is greater. This phenomenon is attributed to the physiology of the cochlea. It is a well-known fact that the apical end of the cochlea where lower frequencies are represented is broader and contains a greater number of rows of hair cells than the basal turn (von Bekesy, 1960; see Zemlin, 1998). This could be the reason that despite differences in the levels of residual hearing, the participants of three groups did not demonstrate significant difference in speech perception performance for speech stimulus filtered at $500 \mathrm{~Hz}$ and $1000 \mathrm{~Hz}$ cut-off frequencies. This is consistent with the research evidence that the degradation is less severe at the lower frequencies than at the higher frequencies although the amount of speech information that can be extracted from an audible signal decrease with increased hearing loss. On an average, an individual with a 100-dB hearing loss at $500 \mathrm{~Hz}$ can extract about half the information available to a normal-hearing individual from the same amount of audible signal (Ching, Psarros, Hill, Dillon \& Incerti, 2001).

There was a significant effect of degree of hearing impairment on speech perception performance for speech stimuli filtered at $1500 \mathrm{~Hz}, 2000 \mathrm{~Hz}, 2500 \mathrm{~Hz}, 3000 \mathrm{~Hz}$ cut-off frequencies. The SIS significantly reduced as a function of the degree of hearing impairment for speech stimuli filtered at $1500 \mathrm{~Hz}, 2000 \mathrm{~Hz}, 2500 \mathrm{~Hz}$, and $3000 \mathrm{~Hz}$. In a typical sensorineural hearing impairment, the damage occurs primarily to the hair cells present in the cochlea. When the hearing impairment is less than severe, the outer hair cells are generally damaged and presumed to have sufficient existing inner hair cells and to accompany neural connections to allow amplified sounds to transmit speech information to the central auditory system (Turner, Gantz, Lowder \& Gfeller, 2005). This could be the reason that the participants with moderate hearing impairment achieved mean SIS that was falling within the normal range. While the participants with moderately-severe and severe hearing impairment did not achieve SIS in the normal range, the participants with moderately-severe hearing impairment achieved significantly higher SIS as compared to participants with severe hearing impairment. As the degree of hearing loss increases, the damage to the hair cells affects not just the more vulnerable outer hair cells, but also the inner hair cells that are responsible to transmit signals to the central auditory system. Hence, the transmission pattern of basilar membrane vibrations in the cochlea to the central auditory system is problematic due to damaged inner hair cells in spite of having an intact auditory nerve (Turner, Gantz, Lowder \& Gfeller, 2005). This could be the possible reason that the participants with moderately severe and severe hearing impairment demonstrated significantly lower SIS as compared to participants with moderate hearing impairment.

In summary, individuals with a severe degree and/or sloping type of hearing impairment are limited in their ability to access a wider range of speech spectrum. Consequently, they experience greater difficulties in perceiving speech sounds whose spectral energy is predominantly dominated in the higher frequency region. Besides, studies have reported negative consequences for speech perception among individuals who have difficulties in perceiving high-frequency speech information (Stelmachowicz et al. 2001; McCreery et al. 2013). The high frequency speech identification tests that are specifically designed to assess individuals who have difficulties in perceiving high frequency speech sounds would therefore be sensitive to identify their perceptual difficulties (Kumar, Varudhini \& Ravichandran, 2016). Furthermore, due to the technical issue of integrating high power and high bandwidth in the same transducer, the output bandwidth of conventional hearing aids is insufficient to produce consistently audible high-frequency speech information. Therefore, audiologists must attempt trails using hearing aids with frequency lowering strategies that are intended to restore high-frequency speech cues that would otherwise be unavailable to individuals with sensorineural hearing impairment (Simpson, Hersbach \& McDermott, 2005).

\section{CONCLUSIONS}

We investigated the effect of filtered speech stimulus on speech perception performance of native speakers of Marathi as a function of the degree of hearing impairment. While speech perception performance improved with an increase in cut-off frequency of speech stimulus, the improvement in speech perception did not correspond with what would be expected with an increase in cut-off frequency of speech stimulus for participants with moderately-severe and severe hearing impairment relative to participants with moderate hearing impairment. The reduction in hearing sensitivity as well as limited ability to access the broader speech spectrum might have reduced the audibility of acoustic cues among participants with moderately-severe and severe hearing impairment. On the other hand, the participants with moderate hearing impairment achieved SIS in the normal range due to less reduction in hearing sensitivity as well as greater ability to access the broader speech spectrum might have increased audibility of relevant acoustic cues. The findings of the present study highlight the need to include high frequency speech identification tests as part of speech perception assessment and attempt trails using hearing aids with frequency lowering strategies in the management of individuals with a greater degree of sensorineural hearing impairment. 


\section{REFERENCES}

[1] Avilala, V. K., Prabhu, P. \& Barman, A. (2010). The Effect of Filtered Speech on Speech Identification Scores of Young Normal Hearing Adults. Journal of All India Institute of Speech and Hearing, 29(1):115-119.

[2] Baer, T., Moore, B. C. J. \& Kluk, K. (2002). Effects of Low-Pass Filtering on the Intelligibility of Speech in Noise for People with and without Dead Regions at High Frequencies. Journal of the Acoustical Society of America, 112:1133-1144.

[3] Bhargavi, C., Prakash, S. G. R., Kumar, S. B. R. \& Sindhura, Y. G. (2010). Development of time-compressed speech test for children between 8-12 years of age in Telugu. Language India, 10:96-115.

[4] Boothroyd, A. (2008). The Acoustic Speech Signal. In: J. R. Madel \& C. Flexer (eds.), Pediatric Audiology. NY: Thieme (pp. 159-167).

[5] Bornstein, S. P., Wilson, R. H. \& Cambron, N. K. (1994). Low-and High-pass Filtered North-western University Auditory Test No. 6 for Monaural and Binaural Evaluation. Journal of American Academy of Audiology, 5:259-264

[6] Ching, T. Y. C., Dillon, H., Katsch, R. \& Byrne, D. (2001). Maximizing Effective Audibility in Hearing Aid Fitting. Ear and Hearing, 22(3):212-224

[7] Ching, T. Y. C., Psarros, C., Hill, M., Dillon, H. \& Incerti, P. (2001). Should Children who use Cochlear Implants Wear Hearing Aids in the Opposite Ear? Ear and Hearing, 22:365-380.

[8] Fu, Q. J., Shannon, R. V. \& Wang, X. (1998). Effects of Noise and Spectral Resolution on Vowel and Consonant Recognition. Acoustic and Electric Hearing. Journal of the Acoustical Society of America, 104:3586-3596.

[9] Hogan, C. A. \& Turner, C. W. (1998). High-frequency Audibility: Benefits for Hearing-impaired Listeners. The Journal of the Acoustical Society of America, 104(1):432-41.

[10] Kumar, S. B. R., Mohanty, P., Ujawane, P. A. \& Huzurbazar, Y. R. (2016). Conventional Speech Identification Test in Marathi for Adults. International Journal of Otorhinolaryngology and Head and Neck Surgery, 2(4):205-215.

[11] Kumar, S. B. R., Patil, P. P., Saxena, U., Bapuji, M. \& Chacko, G. (2021). Effect of Spectrally Modified Speech on Speech Perception of Native Speakers of Marathi. Indian Journal of Otology, 27(2): 63-67.

[12] Kumar, S. B. R., Varudhini, S. K. \& Ravichandran, A. (2016). Speech Identification Test in Telugu: Considerations for Sloping High Frequency Hearing Loss. International Journal of Speech \& Language Pathology and Audiology, 4: 63-73.

[13] Lorenzi, C., Gilbert, G., Carn, H., Garnier, S. \& Moore, B. C. J. (2006). Speech Perception Problems of the Hearing Impaired Reflect Inability to use Temporal Fine Structure. Proceedings of the National Academy of Science: USA, 103(49): 18866-18869.

[14] Mackersie, C. L., Crocker, T. L. \& Davis, R. (2004). Limiting High-Frequency Hearing Aid Gain in Listeners with and without Suspected Cochlear Dead Regions. Journal of the American Academy of Audiology, 15:498-507.

[15] McCreery, R. W., Brennan, M. A., Hoover, B., et al. (2013). Maximizing Audibility and Speech Recognition with Nonlinear Frequency Compression by Estimating Audible Bandwidth. Ear and Hearing, 34(2): e24-e27.

[16] Moore, B. C. J., Glasberg, B. R. \& Baer, T. (1997): A Model for the Prediction of Thresholds, Loudness and Partial Loudness. Journal of Audio Engineering Society, 45:224-240

[17] Pavlovic, C. V. (1984). Use of the Articulation Index for Assessing Residual Auditory Function in Listeners with Sensorineural Hearing Impairment. Journal of Acoustic Society of America, 75: 1253-1258.

[18] Shannon, R. V., Zeng, F. G., Kamath, V., Wygonski, J. \& Ekelid, M. (1995). Speech Recognition with Primarily Temporal Cues. Science, 270(5234):303-304

[19] Simpson, A., Hersbach, A. A. \& McDermott, H. J. (2005). Improvements in Speech Perception with an Experimental Nonlinear Frequency Compression Hearing Device. International Journal of Audiology, 44(5):281-292.

[20] Smith, Z. M., Delgutte, B. \& Oxenham, A. J. (2002). Chimaeric Sounds Reveal Dichotomies in Auditory Perception. Nature, 416(6876):87-90.

[21] Stelmachowicz, P. G., Pittman, A. L., Hoover, B. M., et al. (2001). Effect of Stimulus Bandwidth on the Perception of /s/ in Normal-and Hearing-Impaired Children and Adults. The Journal of the Acoustical Society of America, 110(4):2183-2190.

[22] Stelmachowicz, P. G., Pittman, A. L., Hoover, B. M., Lewis, D. E. \& Moeller, M. P. (2004). The Importance of High Frequency Audibility in the Speech and Language Development of Children with Hearing Loss. Archives of Otolaryngology Head-Neck Surgery, 130:556-562.

[23] Summerfield, A. Q., (1987). Speech Perception in Normal and Impaired Hearing. British Medical Bulletin, 43(4):909-925.

[24] Turner, C. W. \& Henry, B. (2002). Benefits of Amplification for Speech Recognition in Background Noise. Journal of the Acoustical Society of America, 112: 1675-1680.

[25] Turner, C. W., Souza, P. E. \& Forget, L. N. (1995). Use of Temporal Envelope Cues in Speech Recognition by Normal and Hearing-impaired Listeners. Journal of Acoustic Society of America, 97:2568-2576.

[26] Turner, C., Gantz, B., Lowder, M. \& Gfeller, K. (2005). Benefits Seen in Acoustic Hearing Plus Electric Stimulation in Same Ear. The Hearing Journal, 58(11): 53-55.

[27] Vickers, D. A., Baer, T. \& Moore, B. C. J. (2001). Effects of Low-Pass Filtering on Speech Intelligibility for Listeners with Dead Regions at High Frequencies. British Journal of Audiology, 35:148-149.

[28] von Bekesy (1960). Experiments in Hearing, New York: McGraw-Hill.

[29] Winn, M. B., Won, J. H. \& Moon, I. J. (2016). Assessment of Spectral and Temporal Resolution in Cochlear Implant Users using Psychoacoustic Discrimination and Speech Cue Categorization. Ear and Hearing, 37(6):377-390.

[30] Yadav, N., Kumar, S. B. R., Annapurna, S. B. \& Vinila, V. J. (2011). The Effect of Stimulus Bandwidth on Perception of Fricative /s/ among Individuals with Different Degrees of Sensorineural Hearing Loss. Theory and Practice in Language Studies, 1(12): 1679-1687.

[31] Zemlin, W. R. (1998). Speech and Hearing Science. Anatomy and Physiology (4th ed), Boston: Alyn and Bacon. 
S. B. Rathna Kumar has completed M.Sc. (Speech \& Hearing) from All India Institute of Speech \& Hearing, Mysore, India, and Ph.D. (Applied Linguistics) from Center for Applied Linguistics \& Translation Studies, University of Hyderabad, India. Currently Rathna Kumar is a Faculty at AIi Yavar Jung National Institute of Speech \& Hearing Disabilities (Divyangjan), Mumbai, India. His areas of interest are Behavioural Hearing Assessment, Applied Linguistics, and Phonetics.

Niharika Dash is working as Clinical Tutor in the Department of Speech Language Pathology at Institute of Health Sciences, Bhubaneshwar, India. She has completed Bachelor's as well as Master's Degree in Audiology and Speech Language Pathology at Ali Yavar Jung National Institute of Speech \& Hearing Disabilities (Divyangjan), Mumbai, India. She is the Corresponding Author for this research article.

Mendem Bapuji has completed M.A (Linguistics) from Osmania University, and Ph.D. (Applied Linguistics) from Centre for Applied Linguistics and Translation Studies, University of Hyderabad, India. At present, Bapuji is a faculty at MAA Institute of Speech \& Hearing, Hyderabad, India. His areas of interests are Applied Linguistics, Descriptive Linguistics, Language Disorders, Language Contact and Convergence, Language Endangerment Studies, and English Language Teaching and Testing.

S. Arulmozi is an Associate Professor at the Centre for Applied Linguistics and Translation Studies, University of Hyderabad, India. He has also worked as an Assistant Professor in the Department of Linguistics, Dravidian University, India. Arulmozi has completed two major projects, viz. Indian Languages Corpora Initiative (Telugu) and Telugu WordNet. His areas of specialization include Corpus Studies, Language Endangerment Studies, and Sociolinguistics.

Chandrahas Chandanshive has completed B.Sc. (ASR) in 1996 and M.Sc. (ASR) in 2000 from T. N. M. College Under Mumbai University. Currently he is working as Assistant Professor (Audiology \& Speech Language Pathology) in B. Y. L. Nair Charitable Hospital and T. N. M. College, Mumbai, India. He is also pursuing Ph.D. (Allied Health Science) from Maharashtra University of Health Sciences, India. The areas of his interest are pediatric audiology and amplification devices. 